\title{
Estudios etnográficos sobre el desarrollo infantil en comunidades indígenas de América Latina: contribuciones, omisiones y desafíos
}

\author{
Carolina Remorini*
}

\section{Resumen}

En las últimas décadas, ha habido un progreso sustancial en el estudio del Desarrollo Infantil Temprano (DIT) desde perspectivas interdisciplinarias e transculturales. Varios autores reconocen la contribución de la antropología al debate sobre la primera infancia, en diálogo con la psicología, las neurociencias, la educación y la medicina. Estos estudios subrayan la importancia de los contextos ecológicos en lo que se refiere al crecimiento físico, las trayectorias de desarrollo y los efectos sobre la salud a mediano y largo plazo. La Antropología hace hincapié en la noción de que el desarrollo de los niños depende de las interacciones que éstos entablan con su entorno inmediato y las actividades en las que participan. La importancia dada al ambiente desde una perspectiva ecológica reconoce el valor heurístico de los estudios etnográficos. En este trabajo caracterizamos, algunos ejes y modos de abordaje que se destacan en la producción etnográfica sobre infancia y desarrollo infantil en Latinoamérica, especialmente, sobre comunidades indígenas. En segundo lugar, analizamos y discutimos las posibilidades de interface entre la etnografía y las disciplinas que tradicionalmente se han ocupado del desarrollo infantil y reflexionamos sobre sus contribuciones en términos metodológicos a la comprensión de un proceso de carácter multidimensional, evitando los reduccionismos propios de cada mirada disciplinar. En relación a ello, discutimos los desafíos que la etnografía presenta en el contexto de la investigación inter y transdisciplinaria del DIT. Para concluir, reflexionamos sobre la necesidad de recuperar el DIT como objeto de estudio etnográfico, como lo fue en los inicios de la disciplina.

Palabras claves: Desarrollo infantil. Etnografía. América Latina.

* Profesora Adjunto de Etnografía I en Universidad Nacional de La Plata, Argentina. Investigador Asistente Consejo Nacional de Investigaciones Científicas y Técnicas - Argentina. 


\section{Algunas razones para el estudio del Desarrollo en la Primera Infancia}

El Desarrollo Infantil Temprano (DIT) es la etapa del desarrollo humano correspondiente al período de la vida que se extiende desde la concepción a los cinco años. Durante este período se forman del 85 al $90 \%$ de las funciones del sistema nervioso central, los primeros lazos afectivos de cuyo desarrollo dependerán las siguientes relaciones interpersonales y el desempeño social del sujeto y se sientan las bases del desarrollo cognitivo. Desde el punto de vista biomédico y epidemiológico se plantea que en este período el sistema inmunológico del niño madura más rápidamente, al tiempo que los niños se hallan en un estado de mayor vulnerabilidad frente a los agentes causantes de enfermedades infecciosas. Esto significa que determinadas patologías que ocurren en este período pueden impactar en su ulterior desarrollo y salud. Entre ellas, parasitosis, infecciones respiratorias agudas, enfermedades gastrointestinales y anemia por deficiencia de hierro, entre las más frecuentes en el ámbito latinoamericano. Existe también un reconocimiento temprano por parte de las ciencias sociales, la antropología en particular, de la importancia de los eventos y procesos que ocurren en este período que otras disciplinas denominan la "primera infancia", lo que es atestiguado por la abundante literatura etnográfica, especialmente sobre sociedades denominadas "no occidentales". Al respecto, los cuidados brindados a los niños, la calidad de los vínculos con sus cuidadores y otros miembros de su entorno social, los modos de transmisión cultural, los riesgos que en su medio ambiente -social y naturalse presumen para la vida de los nińos y las decisiones que los adultos toman en relación a su salud e integridad, constituyen algunos de los aspectos clave sobre los que se centran las investigaciones etnográficas acerca de este período de la vida (REMORINI, 2009).

Dentro de la primera infancia, el primer año es visto como un período crítico para el desarrollo del niño, lo que justifica la investigación científica interdisciplinaria y la intervención oportuna de las políticas públicas. En este sentido el DIT es actualmente el principal foco de interés de organismos multilaterales y de los gobiernos de diversos países que invierten recursos financieros en el DIT por sus ventajas en términos de la promoción de la salud a escala individual y colectiva, de la mejora del rendimiento educativo y del desarrollo socioeconómico de un país (SHONKOFF; PHILLIPS, 2000; VARGAS BARÓN, 2008; NIKODEM, 2009). 
Otra de las razones por las cuales es necesario focalizar en la primera infancia es el impacto de las primeras experiencias en términos de la trayectoria vital del individuo. Como señala Herztman (2010, p. 32 ), "what happens to children in their earliest years is critical for their development throughout the life course". En este sentido, estudiar lo que sucede en los primeros ańos de vida nos permite entender cómo envejecen los seres humanos en diferentes contextos, problemática de actual relevancia dado los procesos de transición demográfica.

La publicación de From Neurons to neigborhood: the science of early childhood development, estudio financiado por la Academia Nacional de Medicina de Estados Unidos (SHONKOFF; PHILLIPS, 2000), constituye un claro ejemplo de aquello que en el presente constituye el eje de las discusiones actuales en torno al desarrollo infantil temprano. Se parte de la consideración de que "el conocimiento sobre el desarrollo infantil temprano de mayor calidad es siempre multidimensional y transdisciplinario" (SHONKOFF; PHILLIPS, 2000, p.14 ). Este conocimiento se extiende desde esfuerzos para comprender la bioquímica y los circuitos en pleno desarrollo del cerebro hasta largas investigaciones sobre características familiares, influencias del vecindario y de valores culturales que afectan tanto el bienestar de los nińos en la medida en que se van desarrollando. Afirmando a continuación que "Las interacciones entre las ciencias del desarrollo infantil temprano y el diseño de políticas son problemáticas y requieren de un replanteo dramático" (SHONKOFF; PHILLIPS, 2000, p.20).

A partir de este estudio y otros en el mismo sentido, en las últimas décadas se ha avanzado sustancialmente en el estudio del DIT desde la psicología, las neurociencias, las ciencias de la educación, la medicina y también desde la Antropología - específicamente la Etnografía (WEISNER, 1996). Desde estos estudios se plantea la relevancia del ambiente en el que el niño crece y se desarrolla, En este sentido, la importancia otorgada al ambiente -definido desde una perspectiva ecológica (BRONFEBRENNER, 1987) como una compleja constelación de variables psicosociales, biológicas, económicas, políticas, culturales e históricas- otorga vigencia y valor heurístico a los estudios etnográficos.

La Etnografía - en tanto estudio de modos de vida contemporáneos - y las características propias de su metodología -microescala, énfasis en la perspectiva de los actores, privilegio de los procedimientos inductivos y uso oportunista de 
diversas técnicas cuali-cuantitativas, permiten aproximarnos a aspectos de la crianza y del desarrollo de los niños que no son fácilmente accesibles mediante otro tipo de abordajes. Los estudios etnográficos han demostrado extensamente que los sentidos asignados a la infancia así como las experiencias infantiles y las trayectorias a las que dan origen difieren según el contexto en el que los nińos viven y crecen. De modo que el objetivo central de la investigación etnográfica es describir y comprender los ambientes o "escenarios" culturales que influencian esas trayectorias y la vida cotidiana de los sujetos, las herramientas y recursos que los niños utilizan para desarrollarse en cada uno de ellos (WEISNER, 1996; REMORINI, 2009).

No obstante la relevancia de la perspectiva y los datos antropológicos, algunos autores han insistido en que el compromiso de los antropólogos en investigaciones y en el diseño de políticas públicas en torno al DIT dista de ser el adecuado. En un artículo publicado en Anthropology News, Vargas Barón (2008, p. 7 ) plantea que "[...] anthropologist have been 'largely absent' for a long time in the design of policies and decision-making in ECD, despite the centrality of this issue for the emergence of anthropology". Asimismo argumenta, al igual que Shonkoff y Phillips (2000), que es necesario reconsiderar los lazos entre universidades, organizaciones científicas y agencias gubernamentales, y que los datos antropológicos deben estar más accesibles a quienes diseñan políticas públicas, así como a las comunidades estudiadas, a fin de aumentar su participación en el diseño y ejecución de tales políticas, especialmente cuando se trata de temas y problemas que suelen ser abordados desde concepciones y valores universalistas, desconociendo la historia y las prácticas culturales de sociedades concretas.

Teniendo en cuenta estas consideraciones, en este trabajo nos interesa caracterizar algunos ejes y modos de abordaje que se destacan en la producción etnográfica sobre infancia y desarrollo infantil en Latinoamérica, en particular, sobre comunidades indígenas. En segundo lugar, analizar y discutir las posibilidades de interface entre la etnografía y las disciplinas que tradicionalmente se han ocupado del desarrollo infantil temprano, y en base a ello, reflexionar sobre las contribuciones de la etnografía en términos metodológicos, a la comprensión de un proceso de carácter multidimensional, evitando los reduccionismos propios de cada mirada disciplinar. En relación a ello, discutimos los desafíos que la etnografía presenta en el contexto de la investigación inter y transdisciplinaria del DIT. Para concluir, reflexionamos 
sobre la necesidad de recuperar el DIT como objeto de estudio etnográfico, como lo fue en los inicios de la disciplina. En relación a esto último, no es mi intención entrar aquí en un camino bastante transitado en los últimos años, el de la constatación del "vacío etnográfico" en torno a la infancia y niñez (NUNES, 1999, 2003; COHN, 2005; JAMES; JENKS; PROUT, 1998; JENKS, 1996; HIRSCHFIELD, 2002; SZULC, 2006; HECHT, 2004). Lo que intento en este trabajo es plantear la importancia de restituir un espacio en los estudios etnográficos para el abordaje esta etapa de la vida y de los infantes como actores legítimos e interesantes para la investigación antropológica, en virtud de algunas problemáticas definidas desde otros campos del conocimiento y desde las políticas públicas, así como de un interés renovado en la infancia en el seno mismo de la Etnografía.

\section{Desarrollo infantil temprano en perspectiva etnográfica: interrogantes $y$ aportes}

En relación con los señalamientos realizados hasta aquí, emergen dos preguntas a las cuales la etnografía puede ofrecer respuestas a partir del estudio empírico de la crianza y desarrollo de los nińos en diferentes sociedades: ¿A qué llamamos "desarrollo infantil"? y ¿A qué llamamos "ambiente" y desde qué criterios un ambiente es definido como "saludable" o "riesgoso" para el desarrollo infantil?

Consideramos que la primera pregunta es relevante porque frente a la diversidad histórica y cultural de los modos de organizar la infancia y sus etapas y de conceptualizar los procesos de crecimiento y desarrollo, así como de las prácticas de crianza y cuidado de los nińos -tal como lo revelan los estudios etnográficos en sociedades no occidentales primero, y occidentales luego-, aún hoy prevalece desde ciertos enfoques una visión unilineal y universalista del desarrollo y crecimiento infantil.

En cuanto a la segunda, se trata de un aspecto central al momento de analizar las trayectorias de desarrollo infantil en América Latina. En nuestra región las condiciones en las que crecen y se desarrollan los niños indígenas se caracterizan en general por la inadecuación de los servicios de salud y educación, barreras de acceso y problemas de equidad, no sólo para los niños sino para las familias indígenas en general. Los niveles de pobreza más elevados coinciden con las regiones en las que vive población de origen indígena (CEPAL, 2007; 
LORENZETTI, 2007). Esto ha dado lugar a visiones victimizantes de los niños y sus familias, que se asocian a su vez con la idea de marginalidad y vulnerabilidad extrema. En este marco, las caracterizaciones provenientes de los servicios y programas de los Estados o de organismos internacionales focalizan en la carencia que sufren, no sólo material sino también social y moral. Muchas veces se cuestiona la capacidad de los padres y las comunidades para proveer un entorno saludable y adecuado para el desarrollo. Es decir, prevalece una visión negativa sobre el entorno y la crianza de estos niños, que sólo reconoce los déficits y opaca los aspectos positivos de las relaciones sociales y prácticas que se dan en estos contextos. Con estos señalamientos no pretendemos negar los efectos negativos de las condiciones de pobreza y crisis ambiental que afecta a las poblaciones indígenas de America Latina, con su consecuente impacto en los niveles de salud y en lo que se ha denominado transiciones epidemiológicas y nutricionales (POPKIN, 1998). Por el contrario, hemos dado cuenta en forma extensa de estas cuestiones en otros escritos (REMORINI, 2009, 2010a; SY; REMORINI, 2009). Lo que nos interesa subrayar aquí, es que se necesita observar el desarrollo infantil desde perspectivas que recuperen los significados y experiencias de los sujetos en los contextos locales, y que consideren los múltiples factores y procesos que inciden positiva y negativamente en los niveles de desarrollo y salud de los niños indígenas. En este sentido, a lo largo de este trabajo, analizaremos los aportes de la etnografía en términos de "desnaturalizar" y cuestionar aproximaciones normalizadoras y universales de las trayectorias de desarrollo humano.

\section{Crecimiento y Desarrollo: hacia la superación de las dicotomías naturaleza/cultura y universal/particular}

"Crecimiento" y "Desarrollo" son dos conceptos que han definido "las dos funciones esenciales de la vida de un niño" (Archivos Latinoamericanos de Pediatría, no 11, 1906 apud COLANGELO, 2012, p. 52). El crecimiento es explicado como el aumento progresivo de tamańo y masa corporal; el desarrollo, como diferenciación anatómica y funcional, como complejización de la estructura de los órganos y sus funciones. En general, el desarrollo ha sido definido como un proceso evolutivo, que supone el despliegue de funciones cada vez más complejas. Así, una clásica definición es la de René Spitz, quien plantea que el término suele aplicarse a "[...] the appearance of forms, functions 
and behaviors that are the result of exchanges between the organism with its environment" (SPITZ, 1985, p. 18).

Siguiendo a Jenks (1996), lejos de ser la expresión de procesos naturales, "crecimiento" y "desarrollo" responden a un sentido del cambio socialmente construido. Esta autora caracteriza esta construcción en términos de una teoría naturalizada del tránsito social del niño en relación con el mundo adulto, teoría que la antropología y sociología clásica han considerado en términos de "enculturación" o "socialización". En términos críticos, puede afirmarse que los conceptos de crecimiento y desarrollo no sólo se ponen en juego en el ámbito de la medicina, sino que constituyen las dos grandes metáforas a partir de las cuales las sociedades modernas han comprendido y explicado la niñez (COLANGELO, 2012).

Tanto el crecimiento como el desarrollo constituyen para las sociedades occidentales, indicadores de transición entre etapas de la vida. A través de la idea de desarrollo, siguiendo a Colangelo (2012), la nińez es explicada y articulada con la noción de futuro: implica la idea de una temporalidad lineal a lo largo de la cual el nińo debe cambiar, y este cambio es un proceso natural, realización de lo que es biológicamente inherente y esperado (JENKS, 1996).

Las funciones y procesos involucrados en el desarrollo infantil han sido examinados y conceptualizados desde estas disciplinas como "patrones universales" de la especie que caracterizan la "naturaleza infantil" y definen parámetros de "normalidad". A partir de estas conceptualizaciones de la infancia y el desarrollo infantil, la pediatría y la puericultura por un lado, y la psicología y la pedagogía por otro, edificaron la especificidad de sus abordajes en base a la distinción entre crecimiento y desarrollo, como dos dimensiones posibles para el estudio de los niños. Como parte de un intenso proceso de medicalización de la vida en general y de la niñez en particular, a fines del siglo XIX la pediatría se centra en el abordaje clínico y el seguimiento del crecimiento y desarrollo y, vinculada con ella pero también con la higiene, la puericultura emerge como "ciencia de aplicación" orientada a la transmisión y divulgación de métodos de crianza "racionales y científicos" a fin de prevenir posibles "desviaciones" del recorrido esperable y de garantizar la "evolución normal" a lo largo de diferentes sub-etapas (COLANGELO, 2012).

Por otra parte, la antropología física también se ubicó en el marco de esta distinción, abordando los factores que afectan el crecimiento biológico de los humanos y la variabilidad intra e interpoblacional observadas. 
En el caso de la Antropología, el temprano interés por la crianza y el desarrollo infantil se relaciona con la meta general de la disciplina de dar cuenta de la variabilidad inter e intracultural sin renunciar a la posibilidad de hallar pautas universales que den cuenta de la unicidad de la humanidad más allá de las experiencias culturales particulares. Es así que durante sus inicios prevaleció lo que Geertz (1989) denominó la "concepción estratigráfica" del ser humano, cuya unidad biológica y psíquica fue sostenida largo tiempo, y explica en parte, la perspectiva simplista y universalista acerca de las trayectorias de desarrollo humano. En esta línea, la oposición biología/cultura y como derivada de ella, la distinción genotipo/fenotipo, es discutida por Ingold $(2006,2008)$ en varios de sus trabajos. Este autor argumenta que mucho de lo que llamamos "cultura" en el ser humano podría ser considerado "biología" en el sentido de constituir adquisiciones corporales especificas resultado de procesos complejos y multidimensionales a lo largo de nuestra historia como especie y de nuestro desarrollo ontogenético, producto de relaciones especificas entre organismos (no genotipos) y sus ambientes específicos. Así, en Against Human Nature, Ingold (2006, p. 259; 273) plantea que:

[...] human capacities are not genetically specified but emerge within processes of ontogenetic development. Moreover the circumstances of development are continually shaped through human activity. There is consequently no human nature that has escaped the current of history. [...] This does not mean, of course, that a human being can be anything you please. But it does mean that there is no way of describing what human beings are independently of the manifold historical and environmental circumstances in which they become-in which they grow up and live out their lives.

La conceptualización de la "naturaleza infantil" asocia esta etapa de la vida a conceptos como transición, incompletitud y plasticidad, los que coexisten con los de "riesgo" y "vulnerabilidad", los que veremos en este trabajo, tendrán considerable impacto en los desarrollos disciplinares. De este modo, la idea de "maleabilidad" y la necesidad de cuidados específicos, presentes ya desde la obra de Boas (2008), han dado origen a concepciones de la infancia, el desarrollo y la crianza infantil que han prevalecido hasta la actualidad. Boas fue el primero en sostener, frente a la idea de la fijeza de los tipos raciales, que desarrollo y el 
crecimiento están afectados por factores sociales y geográficos, los que pueden alterar los patrones prestablecidos genéticamente. Asimismo, sostuvo que el desarrollo del cerebro en los humanos supone un tiempo más prolongado y por lo tanto, es absolutamente sensible al contexto ambiental. Su trabajo fue pionero en el surgimiento de la psicobiologia del desarrollo, y dos de sus conceptos, "neotenia" y "plasticidad" (flexibilidad adaptativa) fueron centrales para entender sus postulados sobre cómo los factores culturales inciden en el desarrollo infantil.

Por otra parte, los estudios sobre infancia y desarrollo infantil desde una perspectiva culturalista - legado de la Escuela de Cultura y Personalidad priorizaron los factores culturales por sobre los psicológicos individuales y los biológicos, lo que contribuyó a que oposiciones tales como naturaleza-cultura o universal-particular continuaran caracterizando los abordajes teóricos sobre el tema durante largo tiempo (WHITING; EDWARDS, 1997).

Si lo que nos define como humanos es, de acuerdo con Geertz (1989), la diversidad de nuestra experiencia más que su unicidad ¿por qué se continúa pensando -desde algunos enfoques teóricos- el desarrollo ontogenético en términos de parámetros universales o capacidades biológicas "pre-programadas" genotípicamente? Por qué se continúa pensando de manera simplista la relación entre "genotipo" y "fenotipo", retomando la crítica planteada por Ingold?

Así, la Etnografía y la Psicología Cultural demostraron tempranamente que "people are not the same wherever you go" (SHWEDER, 1990). Siguiendo a Sweder (1990, p. 5), "[...] classic general psychology asumes that transcendentally, 'deep down' or 'inside' where the central processing mechanism is located, people are the same (or, alternatively, what gives people "psychic unity" is what makes them all the same "deep down" or "inside")".

Asumir simplemente la unidad psíquica implica ignorar la diversidad de etnoteorías sobre la construcción de la persona, sus componentes y relaciones, la variabilidad bio-psico-cultural de los procesos de crecimiento y desarrollo, de las maneras por las cuales una persona se define como tal en un grupo y deviene parte de él. Y en ello interviene no sólo la cultura sino también el tiempo histórico, que nos permiten reconocer cambios y continuidades también al interior de cada sociedad.

En este punto, fue innovador el planteo de Vigotski (1986) en el campo e la psicología, cuando defendió la necesidad de pasar del "niño eterno" al "niño 
histórico" dejando así planteado el estudio del desarrollo humano como proceso histórico y cultural. En relación a ello cabe la pregunta ¿Qué modelo de niño y de infancia se intenta universalizar desde las disciplinas del desarrollo? ¿De qué manera puede la investigación inter y transdisciplinaria aportar al conocimiento del "niño histórico" y abandonar la idea de niñez a-temporal?

Todas estas consideraciones implican una necesaria problematización, desde una perspectiva etnográfica, de aquellas categorías que algunos discursos disciplinares "naturalizan", y reconociendo que detrás de tales "categorías nativas" también subyacen presupuestos culturales que definen el alcance de las intervenciones sobre la infancia y los niños.

\section{Ambiente y desarrollo infantil}

Respecto de la segunda pregunta que nos planteamos, nos interesa destacar el valor de los abordajes ecológicos del desarrollo humano (BRONFENBRENNER, 1987; WHITING; EDWARDS, 1997; SUPER; HARCKNESS, 1986; WEISNER, 1984, 1996; ROGOFF, 2003; ROGOFF, MORELLI, CHAVAJAY, 2010). Desde esta perspectiva, Super y Harkness (1986, p. 552 ) propusieron el concepto de "nicho de desarrollo" que refiere a un sistema compuesto por tres componentes básicos: "(1) the physical and social settings of the child's everyday life; (2) culturally regulated customs of child care and childrearing; and (3) the parental ethnotheories". Estas últimas refieren a "specific cultural models, rooted in the collective history of the community, that provide a framework for adults to build experience and knowledge that guide their rearing practices" (SUPER; HARKNESS, 1986, p. 552 . Desde esta conceptualización, el desarrollo del niño puede ser visto como una adaptación a tales "nichos" dando lugar a diferentes trayectorias (GREENFIELD, 2003).

En este sentido, numerosos autores han estudiado el desarrollo y los procesos de aprendizaje con relación a la participación de los niños en el marco de actividades rutinarias compartidas con otros miembros de su unidad doméstica. Es decir, la observación y registro de las experiencias e interacciones cotidianas del niño en aquellos ámbitos en que participa en forma frecuente, y la complejización de su participación y compromiso en diferentes tareas y ámbitos de su comunidad han constituido el foco de estudios recientes en el campo de la Antropología y la Psicología Cultural (LAVE, 1995; ROGOFF, 2003, 2007; ROGOFF, MORELLI, CHAVAJAY, 2010; GASKINGS, 2000; WEISNER, 1996). 
No obstante, el análisis del impacto del ambiente en el desarrollo no siempre ha tenido en cuenta una definición amplia de ambiente que de cuenta de los múltiples contextos en los que participan cotidianamente y las relaciones que establecen en cada uno de ellos. Por el contrario gran parte de la investigación en psicología general, psicoanálisis y biomedicina - y de las políticas públicas que se sustentan en tales desarrollos-, se han limitado a las interacciones cuidador-niño (WHO, 2004), e incluso, en muchos de estos estudios, cuidador es sinónimo de madre biológica. En este sentido, pese a que la Antropología mostró tempranamente la relevancia del cuidado infantil múltiple y de la diversidad de relaciones y entornos (settings) para el desarrollo del niño, (MEAD, 1993; WEISNER; GALLIMORE, 1977; WHITING; WHITING, 1975; WHITING; EDWARDS, 1997) la focalización en el binomio madrehijo continúa siendo el eje de numerosos estudios y programas del Estado.

Si bien ya Bowlby (1951 apud WHO, 2004) había advertido la necesidad de analizar las relaciones niño-cuidador en el contexto económico y social más amplio $^{1}$, no es hasta la década de 1970 cuando la Psicología comienza a enfocarse en el ambiente más amplio en el que el desarrollo psico-biológico del niño tiene lugar, y a plantear que las relaciones cuidadores-niños son bidireccionales y recíprocas (BRONFENBRENNER, 1987; BOLWBY, 1976). Estas nuevas perspectivas consideran al niño ya no como receptáculo pasivo, sino como un organismo complejo y competente. El origen del desarrollo psíquico no se encuentra sólo en las características de maduración del organismo ni sólo en la modeladora influencia de estímulos externos, sino en la estructurada interacción entre organismo y medio (NIKODEM, 2008). De este el niño es un participante activo en los encuentros sociales con sus cuidadores y otros sujetos de su medio social, produciéndose un ajuste de conductas entre ambos.

Por último, en la actualidad aún existen aproximaciones que se centran exclusivamente en lo que sucede en el contexto doméstico sin analizar las articulaciones que se dan entre este nivel micro y otros niveles organizacionales, entre las familias y las instituciones del estado $\mathrm{u}$ otras que inciden en las trayectorias de los sujetos (BRONFENBRENNER, 1987). En este punto, algunas estrategias metodológicas propias de la etnografía, nos sitúan en el nivel micro, pero a la vez, nos permiten observar las conexiones entre éste y otros niveles de análisis, lo que resultan fundamental para situar las prácticas de crianza en la complejidad de los escenarios en que tienen lugar, y evaluar los diferentes factores que afectan el desarrollo. 


\section{Los estudios sobre infancia y desarrollo infantil en América Latina en foco}

Las visiones de la infancia como "realización futura más que experiencia presente" (SZULC, 2006; COLANGELO, 2012) han impactado decisivamente no sólo en el abordaje médico sino también en las primeras formulaciones en el campo de la antropología y la sociología, lo que se expresa en las teorías clásicas de la socialización infantil, profundamente cuestionadas en la actualidad (TOREN, 1993; LAVE, 1995; COHN, 2002, 2005, entre otros).

Veremos que en el caso de Sudamérica, las etnografías sobre pueblos indígenas escritas antes de 1950 enfatizan una visión "naturalista" del niño, un modo de concebir la infancia más próxima al "salvajismo" que a la "civilización" (SZULC, 2006), donde los niños son caracterizados desde una visión romántica. Como ejemplo de ello podemos citar los estudios sobre los Guarani de Florestan Fernandes (1966) y Egon Schaden (1945), por nombrar algunos "clásicos"2.

En relación a los estudios recientes en torno al tema, la mayoría de ellos desataca la naturaleza histórica y sociocultural de la infancia, abordando las transformaciones en las conceptualizaciones y prácticas en torno a ella a través del tiempo, conjugando fuentes históricas, etnográficas y sociológicas principalmente. Es preciso remarcar que América Latina es una vasta región que comprende varios países con trayectorias históricas diversas, lo que dificulta hablar de la región como un todo. Asimismo, las tradiciones antropológicas varían tanto entre países, como al interior de cada uno. La diversidad de abordajes y tradiciones académicas se corresponde con la diversidad y heterogeneidad de las experiencias infantiles, por lo que es necesario prestar atención a esto, ya que de lo contrario, corremos el riesgo de no comprender la complejidad de la vida cotidiana y las trayectorias infantiles (COHN, 2005).

Al respecto, el estado de la cuestión presentado por Szulc y Cohn (2012) muestra un campo de investigación relativamente reciente, en proceso de consolidación desde la década de 1990 especialmente enfocado en Argentina y Brasil. Dentro de los estudios sobre la infancia desde diferentes perspectivas, se resalta la contribución de varias investigaciones etnográficas sobre la niñez y la crianza en sociedades indígenas (NUNES, 1999; COHN, 2002; SILVA; MACEDO; NUNES, 2002; HECHT, 2004; SZULC, 2006; REMORINI, 2009, 2010a, 2010b; PIRES, 2010; CODONHO, 2007) que de alguna manera constituyen aportes a la desnaturalización del discurso médico y psicológico sobre 
la crianza al describir y analizar otros modos de construir y experimentar las primeras etapas de la vida, así como otras prácticas de cuidado y educación infantil.

No obstante, a pesar del crecimiento del campo, coincidimos con Gottlieb cuando afirma que incluso en estos estudios, los infantes reciben menos atención que los niños mayores "[...] In effect, the ethnography of infants is still, if you will, in its infancy" (GOTTLIEB, 2000, p.121 ). En concordancia con este planteo, Clarice Cohn ha publicado una síntesis de los desarrollos contemporáneos en Latinoamérica en el campo de la "Antropología de la Infancia", en el que señala las principales líneas de trabajo existentes (COHN, 2005). En este trabajo Cohn resalta que si bien hallamos numerosos trabajos sobre educación, en general tratan sobre los ámbitos formales y la consideración de hasta qué punto se adecuan las propuestas pedagógicas a las realidades de los niños/as, enfocando en los niños/ as en edad escolar. $\mathrm{Al}$ respecto, es preciso notar que, al menos en Sudamérica, una parte importante de las investigaciones sobre niñez ha estado influenciada significativamente por el campo de la Antropología de la Educación, aunque en la actualidad encontramos perspectivas críticas que reclaman espacio para las experiencias formativas que exceden el ámbito de la educación formal.

En este contexto, y como hemos señalado en otro lugar (REMORINI, 2009), la primera infancia, los infantes y sus interacciones sociales han ocupado un lugar subsidiario y hasta implícito en las descripciones etnográficas sobre sociedades "tradicionales". Ellos están de alguna manera presentes en las etnografías clásicas cuando se habla de maternidad, de organización familiar, el parentesco, de ciclo vital o de la división genérica del trabajo, si bien es preciso reconocer que muy pocos estudios etnográficos han tomado como objeto de descripción y análisis la conducta infantil o sobre la vida cotidiana de los infantes. Tal vez es por ello que en general su socialización sea descripta casi exclusivamente a partir de las referencias verbales de los adultos, quienes "trasmiten" conocimientos, pautas y valores útiles para la vida adulta en un proceso que es presentado muchas veces como si ocurriera en forma unidireccional. Así, los trabajos escritos desde esta perspectiva presentan a los niños como seres "incompletos", aún no integrantes plenos de la sociedad y que reproducen lo que los adultos y ancianos les enseñan. Por lo tanto, no revisten mayor interés al momento de describir y analizar la cultura del grupo, a diferencia de lo que pueden informar los discursos y prácticas de los adultos (COHN, 2005; SZULC, 2006; REMORINI, 2009). En este sentido, la atribución de "pasividad", la negación de la "agencia" de los niños pequeńos y 
la imposibilidad de considerarlos como "informantes" en el sentido clásico del término ha contribuido a su invisibilización (GOTTLIEB, 2000).

No obstante algunas omisiones, se ha registrado un notable crecimiento de trabajos que enfocan en las experiencias infantiles en los últimos años. No es posible en este espacio dar cuenta exhaustivamente de los antecedentes y referentes en el campo. Sin embargo, podemos destacar algunos ejes sobre los que se han centrado los estudios recientes en algunas áreas indígenas de Latinoamérica. Sólo por mencionar algunos de ellos, y en función de la posibilidad de identificar aspectos sobre los cuales la etnografía puede brindar una mirada diferente del desarrollo infantil, elegimos los siguientes:

1) representaciones sociales, y específicamente "etnoteorías" sobre el desarrollo infantil, la crianza y las primeras etapas de la vida;

2) vínculos cuidadores niños y agentes involucrados en la crianza y cuidado de los niños;

3) experiencias de educación formal y no formal;

4) participación de los niños en actividades de subsistencia e impacto sobre la trayectoria y modos de aprendizaje infantil.

En relación al primer punto, investigaciones y revisiones críticas sobre abordajes clásicos en torno al "ciclo vital" y el proceso de "enculturación" tanto desde la etnografía, como desde la psicología, la historia y la sociología, resultaron en interesantes aportes en las dos últimas décadas acerca de la diversidad temporo-espacial de las etnoteorías sobre la infancia y el desarrollo infantil, y su impacto sobre las trayectorias de desarrollo en Latinoamérica. Muchos de estos estudios toman como referente las premisas de la Nueva Sociología de la Niñez (JAMES; JENKS; PROUT, 1998) y han construido su marco teórico fundamentalmente a partir de vertientes anglosajonas, no obstante, algunos toman los aportes de la escuela francesa de la "sociologie de l'enfance" (SIROTTA, 2001). Asimismo, las nuevas propuestas se caracterizan especialmente por el intento de superar la vieja dicotomía "nature-nurture" y enfocan en el estudio del desarrollo como un proceso sociohistórico y cultural que da cuenta de los modos diversos de organizar la variabilidad humana (CERVERA; MENDEZ, 2006; CERVERA, 2007; GREENFELD, 2003; ROGOFF, 2007). En este sentido, resulta especialmente productiva la intersección entre Antropología y Psicología Cultural. 
Los estudios llevados a cabo en sociedades indígenas fueron los primeros en mostrar el modo en que las representaciones sociales sobre la persona y sobre las diferentes etapas de la vida son cruciales para entender el significado de las prácticas de crianza. La investigación de Clarice Cohn entre los Xikrin (pueblo Kayapó del Xingú) es un ejemplo en este sentido, ya que sólo pueden entenderse prácticas como la pintura corporal-que ocupa gran parte del tiempo y un rol central en el cuidado infantil- en tanto "prácticas formativas" por su relación con los conceptos y teorías indígenas sobre persona, cuerpo, niñez y educación. Asimismo, que resulta central para entender el papel de la niñez y los niños en esa sociedad (COHN, 2000, 2002).

Nuestra propia investigación sobre comunidades Mbya Guarani de Argentina comenzó como un estudio del "ciclo vital" desde una perspectiva etnográfica más tradicional (de hecho el término ha permanecido en el titulo de la tesis de doctorado) pero se transformó progresivamente en el estudio de la crianza y el desarrollo infantil, revisando las concepciones disciplinares sobre ciclo vital, trayectoria, desarrollo, socialización, entre otras (REMORINI, 2009). En este estudio y publicaciones subsiguientes, abordamos las diversas dimensiones que incluyen los Mbya dentro de las categorías propias de "crecimiento" y "desarrollo", focalizando en las etnoteorías (HARCKNESS; SUPER, 1986) y prácticas de crianza orientadas a los niños hasta tres años, sus actividades cotidianas y sus relaciones con su entorno (natural y social), intentando dar cuenta de los factores ecológicos que inciden en el nicho de desarrollo (REMORINI, 2009, 2010a, 2010b, 2011).

Así, numerosos estudios se han centrado en el modo en que los discursos y prácticas de crianza "conforman" o "construyen" niños saludables y competentes en cada nicho ecológico, aunque desde perspectivas y metodologías diversas (COHN, 2000; REMORINI, 2009, 2010a, 2010b; DE SUREMAIN, 2003; CERVERA; MENDEZ, 2006; CERVERA, 2007). Al respecto podemos citar la investigación desarrollada por María Dolores Cervera y colegas del CINVESTAV (México). En varios artículos, Cervera aborda las "etnoteorías parentales" sobre la niñez y los niños, la relación entre la trayectoria de vida y las concepciones mayas sobre el alma, la vida, la persona, el aprendizaje y la salud, en diálogo con estudios clásicos y recientes acerca de otras sociedades indígenas de México y Guatemala. A modo de ejemplo, podemos mencionar su estudio sobre la construcción cultural Maya del "temperamento" infantil, comparando dos comunidades rurales con diferentes características socioambientales e 
históricas (CERVERA; MENDEZ, 2006). Las autoras discuten la idea según la cual "variations in temperament characteristics are to be expected as a result of biological differences among populations or individual features" (CERVERA; MENDEZ, 2006, p.326 ). Por el contrario, proponen que los padres mayas perciben y guían sus acciones de crianza y cuidado en función de las teorías nativas sobre la infancia y el comportamiento infantil, y mediante ello tienden a reproducir "culturally accepted behavioural phenotypes through the structuring of the developmental niche" (CERVERA; MENDEZ, 2006, p. 326).

Finalmente, el estudio interdisciplinario llevado a cabo por investigadores franceses en países de la región andina sudamericana (Bolivia y Perú) ofrece información relevante sobre las prácticas de crianza y cuidado de la salud de los nińos, superando enfoques clásicos (etnomedicina, etnopediatría) para dar cuenta del impacto de las políticas de salud y la articulación entre saberes y prácticas de origen diverso (DE SUREMAIN, 2003)

Respecto del segundo punto (b), tanto los estudios clásicos como los recientes sobre sociedades indígenas muestran que la participación de diversos sujetos en la crianza y cuidado infantil es notablemente frecuente, tal como fuera planteado por Mead (1993), y Weisner y Gallimore (1977), entre otros. Estudios que utilizan el término "alloparenting" o "multiple caretaking" son una importante vertiente en el campo. Nuevamente los trabajos de Cervera sobre los mayas yucatecos dan cuenta de la importancia de las relaciones poliádicas en la crianza. También para el caso de Argentina, además de nuestra propia investigación sobre los Mbya Guarani (REMORINI, 2009, 2010a, 2010b, 2011) existen interesantes desarrollos desde perspectivas interdisciplinarias que involucran la etnografía, la antropología biológica y la biología en su vertiente evolutiva. Un ejemplo es el trabajo de Claudia Valeggia (2009) y colaboradores entre los Qom (Toba) de la región del Chaco. En su estudio comparativo sobre "alloparenting" y su relación con los niveles de fertilidad en dos comunidades Qom (una rural y una urbana), Valeggia intenta contrastar hipótesis derivadas de la teoría evolutiva, tales como la "Cooperative Breeding Hypothesis" (BLAFFERHRDY, 2005 apud VALLEGGIA, 2009). En base a ello, sostiene que el cuidado infantil proporcionado por personas diferentes de la madre biológica cumple un rol central en los patrones reproductivos de nuestra especie. Variaciones en el cuidado infantil están entonces asociadas con variables demográficas tales como número de mujeres adultas con y sin niños, densidad de la población, patrones de fertilidad y mortalidad, distribución por sexo y edad, entre otros. Otra línea de 
trabajo derivada enfatiza en el contexto ecológico de cada población. Asumiendo que "alloparenting evolved as an adaptive response to the demands of the environment" (VALEGGIA, 2009, p.1 ), la autora analiza las transformaciones en el cuidado infantil que registran pueblos cazadores-recolectores cuando migran a contextos urbanos o cuando las condiciones ecológicas de sus territorios originarios se ven dramáticamente alteradas.

En relación a los puntos (a) y (c) podemos citar dos estudios que se convirtieron inicialmente en referentes para el campo: el estudio ya mencionado de Clarice Cohn (2000) sobre Xikrin-Kayapo y el de Angela Nunes (1999, 2003) sobre los niños Awué Xavante. La principal contribución teórica de ambas investigaciones es que articularon las problemáticas especificas del estudio de la niñez con categorías y conceptos que se hallan actualmente en el centro de los debates antropológicos sobre sociedades amazónicas tales como corporalidad, ritual, construcción de las nociones de tiempo y espacio, juego, entre otras.

Siguiendo con las investigaciones llevadas a cabo en Brasil, un abundante corpus de estudios se centran en asuntos vinculados con la educación y el aprendizaje en contextos diversos, especialmente focalizando en las instituciones educativas formales. Al respecto, podemos citar el trabajo de Myriam Alvares (2004) sobre los Maxacali, un pueblo indígena del sudeste de Brasil, que vincula las ideas de persona y aprendizaje con las experiencias educativas de las que participan los niños tanto en el ámbito de los rituales comunitarios como de la escuela, y el trabajo de Antonella Tassinari (2001), acerca de los Karipuna, localizados al norte del país, centrado en las experiencias escolares y sus transformaciones en el tiempo, y la relación entre ello y las concepciones indígenas sobre niñez y aprendizaje.

Otra línea de trabajo de extenso desarrollo en Brasil es la que conduce Ana María Gomes y colegas de la Universidad Federal de Minas Gerais. Los autores trabajan mayormente los procesos de aprendizaje siguiendo la línea teórica formulada por Lave e Wenger sobre "modos de aprendizaje y coparticipación del niño en el proceso", en contextos definidos como "comunidades de práctica" (SILVA; GOMEZ, 2011).

Por último, cabe mencionar el trabajo de Camila Codonho (2007) centrado en los procesos de aprendizaje pero desde una perspectiva diferente. Se ha focalizado en lo que llama "trasmisión horizontal o entre pares", analizando qué y cómo aprenden los niños cuando están implicados en actividades fuera 
de la observación o intervención de los adultos. Su trabajo es sobre los GalibiMaworno de la Guyana Francesa y analiza las prácticas de circulación de saberes infantiles (sobre el ambiente, la mitología, las pautas de parentesco) en el marco de las reglas de la organización social y política del grupo y mediante la observación de las actividades y modos de circulación de los niños en las aldeas.

En Argentina, también los antropólogos que estudian los niños han enfocado en los procesos de aprendizaje dentro y fuera del ámbito escolar. Asimismo, en los procesos de aprendizaje entre pares en diferentes contextos comunitarios (HECHT, 2004; ENRIZ, 2011; SZULC, 2009). En este marco, el trabajo de Ana Carolina Hecht (2010), centrado sobre los procesos de socialización y cambio lingüístico entre los niños Toba (Qom), vincula las prácticas lingüísticas con los diversos modos de aprendizaje, las ideas sobre infancia, adultez, maduración y procesos de construcción de la identidad étnica en contextos urbanos. Un aspecto que se destaca es el rol que juegan los niños como agentes de socialización lingüística, articulando la conservación de la lengua Qom y la incorporación del espańol, según diversos contextos de habla. Otra investigadora que trabaja dentro del mismo equipo es Noelia Enriz (2011) quien ha analizado las experiencias formativas de los niños Mbya Guarani, especialmente a través de la observación de prácticas en contextos lúdicos, rituales y escolares, aunque tal vez centrado principalmente en nińos en edad escolar.

Finalmente, en relación al último punto, el impacto de la participación de los niños en las actividades de subsistencia en sus trayectorias de desarrollo, numerosos estudios toman como punto de partida la obra de Lave (1995) y su conceptualización del aprendizaje situado. En este marco, los estudios sobre comunidades indígenas latinoamericanas muestran que los niños poseen habilidades adquiridas muy precozmente a través de su participación y mutua coordinación con otros individuos en actividades rutinarias. Asimismo, enfatizan en el rol que juegan estas actividades y relaciones sociales como trazadores y organizadores del desarrollo infantil (GASKINGS, 2000; CERVERA; MENDEZ, 2006; ROGOFF, 2003; ROGOFF, MORELLI, CHAVAJAY, 2010, entre otros). Estos estudios subrayan también el rol activo de los niños en la construcción de tales trayectorias de desarrollo mediante su actividad transformadora sobre el entorno (REMORINI, 2009). De este modo, el concepto de agencia infantil ocupa un lugar destacado en estos estudios.

En relación a este aspecto, en numerosas sociedades indígenas los nińos dedican menos tiempo al juego o las actividades de entretenimiento en 
comparación con las sociedades urbanas. Al mismo tiempo, los cuidadores emplean menos tiempo en jugar con los niños que el que emplean en otras actividades consideradas más "productivas". Es decir que las etnoteorias sobre la infancia y el desarrollo infantil modelan las expectativas que tienen los adultos sobre el tiempo de actividades en las que deben involucrarse los niños de acuerdo con su género y edad. Como se observa a partir de los trabajos de Gaskings (2000), Cervera (2007) y nuestra propia investigación (REMORINI, 2009, 2011), se espera que los nińos colaboren y participen activamente en tareas de subsistencia -incluyendo el cuidad de otros nińos- a edades muy tempranas. El juego es una actividad propia de los infantes pero no de los niños mayores. Asimismo, Gaskings (2000) plantea que de acuerdo a las etnoteorías Maya, no se reconoce el juego como un componente importante para el desarrollo "saludable" de los niños, tal como si sucede en la mayoría de las sociedades urbanas contemporáneas. Estas consideraciones resultan de interés, y justifican el debate surgido a partir del análisis de los contextos etnográficos, ya que aspectos como el trabajo y la contribución de los niños a la subsistencia de los grupos domésticos han sido destacados por su papel en el desarrollo "saludable" de los niños. Desde algunos ámbitos se evalúan estas actividades como negativas o condenables, especialmente desde las disciplinas hegemónicas en el estudio del desarrollo infantil y por las instituciones del Estado y ONGs que ven en ellas la causa del fracaso y deserción escolar, en otras palabras, una actividad que atenta contra otra de valor superior como lo es la educación formal, considerada a su vez, garantía de acceso a oportunidades laborales y mejoras en la calidad de vida.

\section{Palabras finales: el desarrollo infantil temprano como campo transdisciplinario}

In what ways can we ask new questions about children and childhood that move beyond the traditional limits of our discipline, or between subdisciplines? (ANTHROPOLOGY, 2008, p. 4).

Ethnography is the most important method in the study of human development because it ensures that the 'cultural place' will be incorporated into understanding development (WEISNER, 1996, p. 306). 
Teniendo en cuenta el panorama presentado en la sección anterior, aunque, insistimos, dista de ser exhaustivo y completo, podemos reflexionar sobre el aporte de la información proveniente de las investigaciones etnográficas sobre la primera infancia. El valor heurístico de la Etnografía ser deriva fundamentalmente de su perspectiva, escala y metodología, que ofrecen la posibilidad de analizar la crianza y el desarrollo infantil en el contexto de modos de vida particulares. Como señala Weisner (1996), la etnografía sitúa al investigador en condiciones inmejorables para analizar los escenarios donde el crecimiento y desarrollo tiene lugar y las herramientas de que los niños disponen para construir sus trayectorias en dichos escenarios. Fundamentalmente, la observación y el diálogo con el otro, permite "poner en suspenso" sus propias categorías y conceptos, trascender las perspectivas disciplinares para privilegiar la de aquellos que se estudia. La metodología propia de la etnografía coloca al etnógrafo en un rol interesante dentro de los equipos interdisciplinarios, acercando estas perspectivas, que necesariamente deben tenerse en cuenta si se intenta realizar cualquier investigación o intervención en el marco de políticas públicas. En este sentido planteamos que la investigación del DIT no sólo debe ser interdisciplinaria, sino, transdisciplinaria, incorporando las perspectivas de todos los sujetos relevantes al campo.

En este marco se instala la reflexión sobre la necesidad de mayor protagonismo en el estudio del desarrollo infantil, tema que acompañó el surgimiento y consolidación de la Etnografía. Junto a ello, la necesidad de apertura hacia los desarrollos de otras disciplinas y orientaciones teóricas Psicología Cognitiva, Psicología life-span, Psicomotricidad, Historia, Sociología - que presentan interesantes avances en este campo. En este sentido, la etnografía en su diálogo con otras disciplinas puede aportar a la superación de dicotomías del tipo universal/particular o naturaleza/cultura las que resultan, como hemos visto, inapropiadas para entender el desarrollo humano. Asimismo, abre la posibilidad de identificar complementariedades, tensiones y disputas entre diferentes modos de pensar y abordar la infancia y los procesos de desarrollo, implica reconocer la heterogeneidad en el seno de las sociedades y las disciplinas y los supuestos que subyacen a tal heterogeneidad y explican el surgimiento de tensiones y disputas, como señalamos a lo largo de las primeras páginas de este trabajo.

Coincidimos con Weisner (1996) cuando plantea que el entrenamiento de los profesionales de las diversas disciplinas del desarrollo debe incorporar 
como parte fundamental el conocimiento etnográfico. Sin embargo, agregamos, consideramos fundamental también un acercamiento por parte de los etnógrafos al lenguaje y metodologías de otras disciplinas. En este sentido, este autor plantea que el "metodocentrismo"-esto es, el uso exclusivo de un método o de los métodos de una sola disciplina- debe ser evitado en el estudio del desarrollo humano

Por todo lo expuesto, y atendiendo a la diversidad de experiencias infantiles y las complejas problemáticas que atraviesan la vida cotidiana de los niños/as en Latinoamérica, consideramos que los estudios sobre el desarrollo infantil constituyen un tema de interés prioritario para nuestros países y, al mismo tiempo, una excelente oportunidad para debatir en torno a conceptos, hipótesis y metodologías más eficaces para el estudio científico del desarrollo humano y los múltiples contextos que lo afectan.

Finalmente, y atendiendo a lo planteado en este artículo, resulta importante asimismo la consolidación de espacios de debate e intercambio y de difusión de nuestros estudios dentro y fuera de América Latina, evitando al mismo tiempo el "aislamiento" en espacios académicos específicos, ya que nuestras investigaciones dan cuenta también de las particulares perspectivas que tienen los niños sobre problemas que los afectan, sus intereses y demandas desde su lugar como participantes activos de las sociedades contemporáneas.

\section{Notas}

1 "Just as children are absolutely depend on their parents for sustenance, so are parents, especially their mothers dependent on a greater society for economic provision. If a community values its children, it must cherish their parents". (BOWLBY, 1951, p. 84 apud WHO, 2004).

${ }^{2} \mathrm{Si}$ bien hay otros ejemplos, elijo este pueblo y estos autores dado que es la literatura que mejor conozco debido a mi tema de investigación.

\section{REFERENCIAS}

ALVARES, Myriam. Kitoko Maxakali: a criança indígena e os processos de formação, aprendizagem e escolarização. Anthropológicas, Recife, v. 15, n. 1, p. 49-78, 2004. 
ANTHROPOLOGY News. Transforming the anthropology of childhood. Arlington, VA, apr. 2008. Disponible en: <http://aaanet.org/pdf/upload/494-Bock-Gaskins-Lancy-In-Focus.pdf>.

BOAS, Franz. Plasticity in child development. In: LEVINE, R. R. Anthropology and child development: a cross-cultural reader. Oxford: Blackwell Publishing. 2008.

BOWLBY, John. El vínculo afectivo. Buenos Aires: Paidós, 1976.

BRONFENBRENNER, Urie. La ecología del desarrollo humano: experimentos en entornos naturales y diseñados. Barcelona: Paidós, 1987.

CEPAL. Políticas y Programas de Salud de Pueblos Indígenas en América Latina. In: CEPAL. Panorama Social de América Latina: 2007. Santiago, 2007. Disponible en: <http://www.eclac.cl/publicaciones/xml/5/30305/ PSE2007_Cap5_AgendaSocial.pdf>. Acceso en: 23 nov. 2007.

CERVERA, Maria Dolores. Etnoteorías parentales, alma y enfermedades infantiles entre los mayas de Yucatán. In: CERECEDO, Magalí Civera; BAUTISTA, Martha Rebeca Herrera (Ed.). Estudios de Antropología Biológica. México, DF: Universidad Autónoma de México, 2007. 13v.

CERVERA, Maria Dolores; MÉNDEZ, R.M. Temperament and ecological context among Yucatec Mayan children. International Journal of Behavioral Development, n. 30, p. 326-337. 2006.

COLANGELO, María A. La crianza en disputa: medicalización del cuidado infantil en la argentina, entre 1890 y 1930. 2012. Tesis (Doctorado en Ciencias Naturales) - Universidad Nacional de La Plata, La Plata, 2012.

COHN, Clarice. Crescendo como um Xikrim: uma análise da infância e do desenvolvimento infantil entre os Kayapó-Xikrim do Bacajá. Revista de Antropologia, São Paulo, v. 43, n. 2, p. 195-222, 2000.

COHN, Clarice. A experiência da infância e o aprendizado entre os Xikrin. In: SILVA, Aracy Lopes da; MACEDO, Ana Vera; NUNES, Angela (Org.). Crianças indígenas: ensaios antropológicos. São Paulo: Global, 2002.

COHN, Clarice. Antropologia da Criança. Rio de Janeiro: Jorge Zahar, 2005. 
CONDONHO, Camila. Aprendendo entre pares: a transmissão horizontal de saberes entre as crianças indígenas Galibi-Marworno (Amapá, Brasil). 2007. 133f. Dissertação (Mestrado em Antropologia Social) - Universidade Federal de Santa Catarina. Florianópolis, SC, 2007.

DE SUREMAIN, Charles-Édouard et al. Miradas cruzadas en el niño: un enfoque interdisciplinario para la salud, el crecimiento y el desarrollo del niño en Bolivia y Perú. Lima: IFEA, 2003.

ENRIZ, Noelia. Jajeroky: un abordaje de la cotidianidad de los niños y niñas mbyá. In: NOVARO, Gabriela (Ed.) Educación y escolaridad en contextos interculturales: temas de investigación, políticas educativas y demandas sociales. Buenos Aires: Editorial Biblos, 2011. p. 103-120.

FERNANDES, Florestan. Notas sobre a educaçâo na sociedade Tupinambá. In: FERNANDES, Florestan. Educação e Sociedade no Brasil. São Paulo: Dominus; Edusp, 1966.

GASKINGS, Suzanne. Children's daily activities in a Mayan Village: a culturally grounded description. Cross-Cultural Research, Lanham, MD, v. 34, n. 375, 2000.

GEERTZ, Clifford. El impacto del concepto de cultura en el concepto del hombre. In: GEERTZ, Clifford. La interpretación de las culturas. Barcelona: Gedisa, 1989.

GOTTLIEB, A. Where have all the babies gone? Toward an anthropology of Infants (and their caretakers). Anthropological Quarterly, Baltimore, MD, v. 73, n. 3, p. 121-132, 2000.

GREENFIELD, Patricia M. et al. Cultural Pathways through universal development. Annual Review of Psycholoy, Palo Alto, CA, v. 54, p. 461-490, 2003.

HARCKNESS, Sarah; SUPER, Charles. The developmental niche: a conceptualization at the interface of child and culture. International Journal of Behavioral Development, New York, n. 9, p. 545-569, 1986. 
HECHT, Ana Carolina. Hacia una revisión de la categoría 'niño' y 'cultura wichî' a través de la escuela en el departamento Ramón Lista (Formosa). In: CONGRESO ARGENTINO DE ANTROPOLOGÍA SOCIAL, 7., 2004, Córdoba. Actas... Córdoba: Universidad Nacional de Córdoba, 2004. 1 CD ROM.

HECHT, Ana Carolina. "Todavía no se hallaron hablar en idioma": procesos de socialización lingüística de los niños en el barrio toba de Derqui (Argentina). Múnich: LINCOM EUROPA, 2010.

HERTZMAN, Clyde. Social geography of developmental health in the early years. Healthcare Quarterly, v. 13, special issue, p. 32-40, 2010.

HIRSCHFELD, Lawrence. Why don't anthropologist like children? American Anthropologist, Malden, MA, v. 104, n. 2, 2002.

INGOLD, Tim. Against human nature. In: AERTS, D.; GONTIER, N.; VAN BENDEGEM. J. P. (Ed.) Evolutionary epistemology, language and culture. Dordrecht: Springer, 2006. p. 259-281.

INGOLD, Tim. Tres en uno: Cómo disolver las distinciones cuerpo, mente y cultura. In: SÁNCHEZ-CRIADO, Tomás (Org.). Tecnogénesis: la construcción técnica de las ecologías humanas. Madrid: AIBR, 2008. 2.v.

JAMES, Allison; JENKS, Chris; PROUT, Alan. Theorizing childhood. Cambridge: Pility Press, 1998.

JENKS, Chris. Childhood. London: Routledge, 1996.

LAVE, Jean. Cognition in practice: mind, mathematics and culture in everyday life. New York: Cambridge University Press, 1995.

LORENZETTI, Mariana. Del "capital social" a la "culturalización de la pobreza”: la construcción de alteridad en los programas de salud para la población indígena. In: JORNADAS DE JÓVENES IVESTIGADORES, 4., 2007, Buenos Aires. Anais electrónicos... Buenos Aires: Universidad de Buenos Aires, 2007. Disponible en: <http://webiigg.sociales.uba. ar/iigg/jovenes_investigadores/4jornadasjovenes/EJES/Eje\%201\%20 Identidades\%20Alteridades/Ponencias/LORENZETTI,\%20Mariana.pdf>.

MEAD, Margaret. Adolescencia, sexo y cultura en Samoa. Barcelona: Planeta Agostini, 1993. 
NIKODEM, María R. Niños de Alto riesgo: intervenciones tempranas en el desarrollo y la salud infantil. Buenos Aires: Paidós, 2009.

NUNES, Angela. A sociedade das crianças A 'uwê Xavante: por uma antropologia da criança. 1999. Dissertação (Mestrado em Antorpologia Social) - Universidade de SãoPaulo. São Paulo, 1999.

NUNES, Angela. Brincando de ser criança: contribuiçôes da etnologia indígena brasileira à antropologia da infância. 2003. Tese (Doutorado em Antropologia da Educação) - Instituto Superior das Ciências do Trabalho e da Empresa. Lisboa, 2003.

PIRES, Flávia. O que as crianças podem fazer pela antropologia? Horizontes Antropológicos, Porto Alegre, v. 16, n. 34, p. 137-157, jul./dez. 2010.

POPKIN, B. M. The nutrition transition and its implications in lowerincome countries. Public Health Nutrition, Cambridge, n. 1, p. 5-21. 1998.

REMORINI, Carolina. Aporte a la caracterización etnográfica de los procesos de salud: enfermedad en las primeras etapas del ciclo vital, en comunidades Mbya-Guaraní de Misiones, República Argentina. 2009. 423f. Tesis (Doctorado en Ciencias Naturales) - Universidad Nacional de La Plata. La Plata, 2009.

REMORINI, Carolina. ¿De qué y por qué se enferman los niños Mbya? Representaciones y prácticas en torno a la salud en contextos socioculturales y ecológicos vulnerables. In: DROVETTA, Raquel; RODRÍGUEZ, Maria Laura. (Org.) Padecimientos en grupos vulnerable del interior de Argentina: procesos históricos y actuales de salud, enfermedad y atención. Córdoba: Ferreyra Editores, 2010a. p. 145-182.

REMORINI, Carolina. Crecer en movimiento: abordaje etnográfico de la crianza y el desarrollo infantil en comunidades Mbya (Misiones, Argentina). Revista Latinoamericana de Ciencias Sociales sobre Niñez y Juventud, Colombia, v. 8, n. 2, jul./dic., p. 961-980, 2010b.

REMORINI, Carolina. Hermanos que cuidan, hermanos que enseñan: trayectorias de desarrollo en perspectiva ecológica. In: CONGRESO ARGENTINO DE ANTROPOLOGÍA SOCIAL, 10., Buenos Aires, 2011. Actas on line... Buenos Aires: Universidad de Buenos Aires, 2011. Disponible en: <http://www.xcaas.org.ar/actas.php>. 
ROGOFF, Bárbara et al. Firsthand learning through intent participation. Annual Review of Psychology, Palo Alto, CA, v. 54, n. 1, p. 175-203, 2003.

ROGOFF, Bárbara et al. Children's development of cultural repertoires through participation in everyday routines and practices. In: GRUSEC, J.; HASTINGS, P. (Ed.) Handbook of Socialization. New York: Guilford, 2007. p 490-515.

ROGOFF, Bárbara; MORELLI, Gilda; CHAVAJAY, Pablo. Chilren's integration in communities and segregation from people of different ages. Perspectives on Psychological Science, Santa Cruz, CA, v. 5, n. 4, p. 431-440, 2010.

SCHADEN, Egon. Educação e magia nas cerimônias de iniciação. Revista Brasileira de Estudos Pedagógicos, Rio de Janeiro, v. 3, n. 8, p. 271-274, 1945.

SHONKOFF, Jack P.; PHILLIPS, Deborah A. (Org.) From neurons to neighborhood: the science of early childhood development. Washington: National Academy Press, 2000.

SHWEDER, Richard A. Cultural psychology: what is it? In: STIGLER, James W.; SHWEDER, Richard A.; HERDT, Gilbert (Org.). Cultural psychology: essays in comparative human development. New York: Cambridge University Press, 1990. p. 1-43.

SILVA, Aracy Lopes da; MACEDO, Ana Vera; NUNES, Angela (Org.). Crianças indígenas: ensaios antropológicos. São Paulo: Global, 2002.

SILVA, Rogerio Correia da; GOMEZ, Ana Maria. Circulando com os meninos: infância, participação e aprendizagens de meninos indígenas Xakriabá. In: CONGRESO ARGENTINO DE ANTROPOLOGÍA SOCIAL, 10., Buenos Aires, 2011. Actas on line... Buenos Aires: Universidad de Buenos Aires, 2011. Disponible en: <http://www.xcaas.org. ar/actas.php>.

SIROTA, Regine. Emergência de uma sociologia da infância: evolução do objeto e do ollhar. Cadernos de Pesquisa, São Paulo, n. 112, p. 7-31, 2001.

SPITZ, René. El primer año de vida del niño. México, DF: Fondo de Cultura Económica, 1998. 
SY, Anahi.; REMORINI, Carolina. Hacia un abordaje integral e intercultural de la salud de los niños Mbya: contribuciones de la investigación etnográfica y desafíos para la gestión pública. In: NOCETI, M. B. (Org.). Oportunidades: caminos hacia la protección integral de los Derechos del niño. Bahía Blanca: Libros en Colectivo, 2009.

SZULC, Andrea. Antropología y niñez: de la omisión a las 'culturas infantiles'. In: WILDE, G.; SCHAMBER, P. (Ed.). Cultura, comunidades y procesos contemporáneos. Buenos Aires: SB, 2006. (Colección Paradigma indicial).

SZULC, Andrea; COHN, Clarice. Anthropology and childhood in South America: perspectives from Brazil and Argentina. AnthropoChildren, Liège, n. 1, 2012.

TASSINARI, Antonella. Da civilização à tradição: os projetos de escola entre os índios do Uaçá. In: SILVA, Aaracy Lopes; FERREIRA, Mariana Kawal Leal (Ed.). Antropologia, História e Educação: a questão indígena e a escola. São Paulo: Global, 2001. p. 175-195.

TOREN, Christina. Making history: the significance of childhood cognition for a comparative anthropology of mind. Man, London, v. 28, n. 3, p. 461478, 1993.

VALLEGIA, Claudia. Changing times for the argentine toba: who cares for the baby now? In: BENTLEY, G.; MACE, R. (Ed.). Alloparenting in human societies: the biosocial society symposium series. Oxford: Berhahn Books. 2009.

VARGAS BARÓN, Emily. Anthropology and early childhood development: a call to action! Anthropology News, Arlington, VA, p. 6-7, apr. 2008.

VIGOTSKI, Lev S. El desarrollo de los procesos psicológicos superiores. Barcelona: Crítica, 2000.

WEISNER, Thomas. Ecocultural niches of middle childhood: a crosscultural perspective. In: COLLINS, A. (Org.) Development during middle childhood. Washington: National Academy Press, 1984. 
WEISNER, Thomas. Why ethnography should be the most important method in the study of human development. In: JESSOR, R.; COLBY A.; SHWEDER, R. (Ed.) Ethnography and human development. Chicago: Universtity of Chicago Press, 1996. p. 305-324.

WEISNER, Thomas; GALLIMORE, Ronald. My brother's keeper: child and sibling caretaking. Current Anthropology, Chicago, v. 18, n. 2, p. 169180, jun. 1977.

WHITING, Beatrice; EDWARDS, Caroline. Children of different worlds: the formation of social behavior. Cambridge: Harvard University Press, 1997.

WHITING, Beatrice; WHITING, John. Children of six cultures: a psicocultural analysis. Harvard: Harvard University Press, 1975.

WHO. World Health Organization. The importance of caregiver-child interactions for the survival and healthy development of young children: a review. Geneve: WHO, 2004. 


\section{Estudos etnográficos sobre o desenvolvimento infantil em comunidades indígenas na América Latina: contribuições, omissões e desafios}

\section{Resumo}

Nas últimas décadas houve um progresso substancial no estudo do Desenvolvimento Infantil Precoce (DIP) a partir de perspectivas interdisciplinares e interculturais. Vários autores reconhecem a contribuição da antropologia ao debate sobre a primeira infância, em diálogo com a psicologia, as neurociências, a educação e a medicina. Estes estudos sublinham a importância dos contextos ecológicos no que se refere ao crescimento físico, às trajetórias de desenvolvimento e aos efeitos sobre a saúde a médio e longo prazo. A Antropologia enfatiza a noção de que o desenvolvimento das crianças depende das interaçóes que estes entabulam com seu entorno imediato e as atividades em que participam. A importância dada ao ambiente desde uma perspectiva ecológica reconhece o valor heurístico dos estudos etnográficos. Neste trabalho, são caracterizados, em primeiro lugar, alguns eixos e modos de abordagem que se destacam na produção etnográfica sobre infância e desenvolvimento infantil na América Latina, especialmente em relação às comunidades indígenas. Em segundo lugar, analisam-se e discutemse as possibilidades de interface entre
Ethnographic studies about child development in Latin American indigenous communities: contributions, omissions and challenges

\begin{abstract}
TIn recent decades, there has been substantial progress in the study of Early Childhood Development (ECD) from interdisciplinary and cross-cultural perspectives. Several authors acknowledge the contribution of Anthropology to the debate about ECD, in dialogue with psychology, neuroscience, education and medicine. These studies stress the importance of ecological contexts in regard to physical growth and later health issues. Anthropology emphasizes the notion that children's development is driven by the interactions they have with those in their immediate environments and the activities in which children participate. In this sense, the importance given to the environment from an ecological perspective recognises the heuristic value of ethnographic studies. In this paper we characterize, first, the existing ethnographic production about infancy and early childhood development in Latin America, focussing on indigenous societies. Second, we analyze and discuss the possibilities of ethnography and interface between disciplines that traditionally have dealt with child development and reflect on
\end{abstract}


a etnografia e as disciplinas que tradicionalmente têm se ocupado do desenvolvimento infantil, e reflete-se sobre suas contribuiçóes em termos metodológicos para a compreensão de um processo de caráter multidimensional, evitando os reducionismos próprios de cada olhar disciplinar. Em relação a isso, sáo discutidos os desafios que a etnografia apresenta no contexto da investigação inter e transdisciplinar do Desenvolvimento Infantil Precoce. Para concluir, reflete-se sobre a necessidade de recuperar o DIP como objeto de estudo etnográfico como ocorreu nos primórdios dessa disciplina.

Palavras-chave: Desenvolvimento infantil. Etnografia. América Latina. their methodological contributions to the understanding of a multidimensional process, avoiding reductionism of each discipline. In relation to this, we discuss the challenges that ethnography face in the context of interdisciplinary research. To conclude, we would reflect on the need to recover the ECD as an object of ethnographic study, as it was in the beginning of the discipline.

Keywords: Child development. Ethnography. Latin America.

\section{Carolina Remorini}

E-mail: carolina.remorini@gmail.com

Recebido em: 7/5/2013

Versáo final recebida em: 25/7/2013

Aprovado em: 29/7/2013 